\title{
Lattice Strain and Band Offsets Determination in ZnSe/ZnS Short-Period Superlattices Grown by MOVPE on (100)GaAs
}

\author{
P. Prete ${ }^{1}$, N. Lovergine ${ }^{2}$, R. Cingolani ${ }^{2}$ and A.M. Mancini ${ }^{2}$ \\ ${ }^{1}$ Istituto per lo studio di nuovi Materiali per l'Elettronica (IME) del CNR, Lecce, Italy \\ ${ }^{2}$ Istituto Nazionale per la Fisica della Materia (INFM), and Dipartimento di Scienza dei Materiali, \\ Università di Lecce, Lecce, Italy
}

Keywords: ZnSe/ZnS Superlattice, Strain, Band Offsets, Electronic States

\begin{abstract}
We report a detailed calculation of the effects of residual lattice and thermal strains on the electronic states of nearly pseudomorphic $\mathrm{ZnSe} / \mathrm{ZnS}$ short-period superlattices (SLs). Comparison is made with experimental electronic transitions derived from $10 \mathrm{~K}$ absorption spectroscopy measurements performed on 30 periods $\mathrm{ZnSe} / \mathrm{ZnS}$ SLs, having well and barrier thickness $\mathrm{L}_{\mathrm{w}}=1.4$ $\mathrm{nm}$ and $\mathrm{L}_{\mathrm{b}}=5.7 \mathrm{~nm}$, respectively. ZnSe/ZnS SLs were grown by metalorganic vapour phase epitaxy (MOVPE) on a $500 \mathrm{~nm}$ thick $\mathrm{ZnS}$ buffer layer and capped with a $210 \mathrm{~nm}$ thick $\mathrm{ZnS}$ epilayer. The whole structures were deposited on $(100) \mathrm{GaAs}$. The intrinsic conduction to valence band offset ratio is finally found to be 8:92 for the above SL structure. The impact of the weak electron confinement on the SLs optical and electronic properties, with special attention to laser applications, is also discussed.
\end{abstract}

\section{Introduction}

ZnSe/ZnS SLs have demonstrated both spontaneous and photo-pumped stimulated emissions in the near-UV region of the visible light [1]. A precise determination of the band offsets between $\mathrm{ZnS}$ and $\mathrm{ZnSe}$ and of the SL electronic properties is possible by comparing what expected on the base of a Kronig-Penney model calculations and the results of $10 \mathrm{~K}$ absorption measurements. However, this requires to carefully consider the actual strain occurring in the heterostructure for which the comparison with experimental data is made. In fact, even for a fully pseudomorphic $\mathrm{ZnSe} / \mathrm{ZnS} \mathrm{SL}$, the $-4.6 \%$ lattice mismatch between $\mathrm{ZnSe}$ and $\mathrm{ZnS}$ introduces a strain value that can be effectively altered by the occurrence of additional thermal deformations originated by the different thermal expansion coefficients of the SL materials, the buffer and the substrate. The overall strain thus distributes inside both $\mathrm{ZnS}$ barriers and $\mathrm{ZnSe}$ wells altering the effective band offsets and need to be taken into account for correct calculations.

\section{Experimental}

Short period $\mathrm{ZnSe} / \mathrm{ZnS}$ MQW heterostructures were grown on (100)-oriented LEC-grown semiinsulating GaAs substrates by low pressure metal-organic vapour phase epitaxy (MOVPE) in an Aixtron AIX 200RD horizontal chamber reactor. The details of the MOVPE growth of the $\mathrm{ZnSe}$ and $\mathrm{ZnS}$ epilayers are reported elsewhere [2]. On a (100) GaAs substrate a $500 \mathrm{~nm}$ thick $\mathrm{ZnS}$ buffer layer was grown at $342^{\circ} \mathrm{C}$, followed by 30 periods $\mathrm{ZnSe} / \mathrm{ZnS} \mathrm{MQW}$ grown at $315^{\circ} \mathrm{C}$, the well width $\left(\mathrm{L}_{\mathrm{w}}\right)$ being equal to $5 \mathrm{ML}(1.4 \mathrm{~nm})$, while the barrier width $\left(\mathrm{L}_{\mathrm{b}}\right)$ being of about $21 \mathrm{ML},(5.7 \mathrm{~nm})$. A growth interruption time of $20 \mathrm{sec}$ was used at each heterointerface, to obtain sharp interfaces. Finally, a $210 \mathrm{~nm}$ thick $\mathrm{ZnS}$ cladding layer was grown on top of the structure at the same growth temperature used for the SLs.

Optical absorption measurements were performed at $10 \mathrm{~K}$ on the mechano-chemically thinned MQW samples prepared following the procedure described elsewhere [3]. The mechanically chopped light of a tungsten halogen lamp was focused onto the sample mounted on the cold finger 
of a closed-circle He cryostat. The transmitted beam was dispersed and detected by a double monochromator $(0.85 \mathrm{~m})$ equipped with a photon counting detector.

\section{Results and discussion}

The excellent optical quality of the MQW samples is confirmed by the sharp excitonic peaks of the absorption spectrum shown in Fig. 1. Also, a clear step-like density of states with superimposed excitonic resonances appears. As the SL total thickness $(213 \mathrm{~nm})$ is bigger than the critical thickness expected for the plastic relaxation at the buffer interface, the SLs will not be completely pseudomorphic to the $\mathrm{ZnS}$ buffer. Thus, in order to correctly evaluate the excitonic transitions and the band offsets, the exact strain of the structure was determined. First, as cathodoluminescence images (not reported here) do not showed misfit dislocations at the $\mathrm{ZnS}$ cap layer/SL interface for the above sample, the cap layer was assumed to have exactly the same lattice parameter than that of the whole SL. The strain state of the $\mathrm{ZnS}$ cap layer has been thus measured by ion-channeling Rutherford backscattering spectrometry at room temperature (RT) and turned out to be $+0.19 \%$. From this measured strain value the in plane lattice parameter $\left(a^{\prime \prime}{ }_{\mathrm{ZnS}}\right)$ of the $\mathrm{ZnS}$ cap layer can be determined, according to the lattice strain definition:

$$
\varepsilon_{\text {res } / Z n S}=\frac{a_{Z n S}^{\prime \prime}-a_{Z n S}}{a_{Z n S}}
$$

and we use the value of $a^{\prime \prime}{ }_{Z n S}=\mathrm{a}^{\prime \prime}{ }_{M Q W}$ to calculate the residual strain of the MQW structure at $300 \mathrm{~K}$ :

$$
\varepsilon_{M Q W}(300 K)=\frac{a_{Z n S}^{\prime \prime}-a_{M Q W}}{a_{M Q W}}=-0.612 \%
$$

with the free-standing lattice constant of the $\mathrm{ZnSe} / \mathrm{ZnS}$ SL expressed by [4]:

$$
a_{M Q W}=\frac{a_{Z n S} G_{Z n S} d_{Z n S}+a_{Z n S e} G_{Z n S e} d_{Z n S e}}{G_{Z n S e} d_{Z n S e}+G_{Z n S} d_{Z n S}}
$$

where $d$ is the thickness of each layer, $a$ is the material lattice constant and $G$ is the shear modulus given by:

$$
G=2 \cdot\left[C_{11}+C_{12}-\frac{2\left(C_{12}\right)^{2}}{C_{11}}\right]
$$

with $\mathrm{C}_{11}$ and $\mathrm{C}_{12}$ elastic stiffness constants. By using Eqs. 2-4 and the materials parameters listed in Tab. 1, the misfit $f_{M Q W / Z n S}$ between the MQW and the $\mathrm{ZnS}$ buffer layer at $300 \mathrm{~K}$ can also be calculated:

$$
f_{M Q W / Z n S}=\frac{a_{Z n S b u f f e r}-a_{M Q W}}{a_{M Q W}}=-0.80 \%
$$

It should be noted that, by cooling down the whole structure from RT to $10 \mathrm{~K}$ for the absorption measurements, a thermal strain contribution $\Delta f^{\text {ther }}{ }_{M Q W / Z n S / G a A s}$ adds to the residual SLs strain derived in Eq. 2 from RBS measurements. It can be demonstrated that this additional thermal strain is given by the relationship:

$$
\Delta f_{M Q W / Z n S / G a A s}^{\text {ther }}=\left[1+\varepsilon_{Z n S / G a A s}^{r e s}\right] \times \Delta f_{M Q W / Z n S}^{\text {ther }}\left(T_{m}\right)+\frac{a_{Z n S}\left(T_{m}\right)}{a_{M Q W}\left(T_{m}\right)} \Delta f_{Z n S / G a A s}^{\text {ther }}
$$

where $\Delta f^{\text {her }}{ }_{M Q W / Z n S}$ indicates the thermal mismatch between the MQW and the ZnS buffer calculated between $300 \mathrm{~K}$ and $\mathrm{T}_{\mathrm{m}}=10 \mathrm{~K}, \Delta f^{\text {her }}{ }_{Z n S / G a A s}$ is the analogous thermal mismatch between $\mathrm{ZnS}$ and GaAs and $\varepsilon_{\text {res, ZnS/GaAs }}$ represents the RT residual lattice strain of the $\mathrm{ZnS}$ buffer layer. In our sample $\varepsilon_{\text {res, ZnS/GaAs }}=0$ because the $500 \mathrm{~nm}$ thick $\mathrm{ZnS}$ buffer is almost totally relaxed [6]. Under these conditions the value of $\Delta f_{\text {ther, } M Q W / Z n S / G a A s}$ turns out to be $+5.55 \times 10^{-4}$ and needs to be added to the lattice residual strain given by Eq. 2 to get the total MQW strain at $10 \mathrm{~K}$, that equals $-5.57 \times 10^{-3}$. From the above strain value the in-plane lattice parameter of the SLs at $10 \mathrm{~K}$ can be calculated 
according to Eq. 2, which finally allows us to estimate the effective strains of wells and barriers. Thus, the value of strain in the $\mathrm{ZnSe}$ wells is equal to $-4.32 \times 10^{-2}$ and it amounts to $+2.29 \times 10^{-3}$ in the $\mathrm{ZnS}$ barriers, indicating that the wells suffer a compressive strain and the barriers a tensile strain. These strain values have been included in the Kronig-Penney calculations of the energy transitions for the SL structure. The absorption transitions were calculated as a function of the conduction band discontinuity. In this calculation it was included both the mass nonparabolicity and the band shift induced by the compressive strain in the well and the weak tensile strain in the barriers. The exciton binding energy was assumed to be in the $30-40 \mathrm{meV}$ range [7]. The presence of the elastic strain causes a hydrostatic and uniaxial deformation inside the structure inducing shifts of the conduction band $(\mathrm{CB})$, hh and $\mathrm{lh}$ band edges [8]. The total deformation potential $\alpha$ has been shared between the $\mathrm{CB}$ and the VB in the same proportion of the band offset.

Table 1: List of materials parameters used in the calculations.

\begin{tabular}{|c|c|c|}
\hline Materials & $\mathrm{ZnSe}$ & $\mathrm{ZnS}$ \\
\hline $\mathrm{a}_{300 \mathrm{~K}}(\AA)$ & $5.6687^{(a)}$ & $5.4060^{(a)}$ \\
\hline $\mathrm{a}_{10 \mathrm{~K}}(\AA)$ & $5.6610^{(\mathrm{b})}$ & $5.4041^{(a)}$ \\
\hline $\mathrm{C}_{11}\left(\mathrm{~N} / \mathrm{m}^{2}\right)$ & $8.26 \times 10^{10(\mathrm{c})}$ & $10.67 \times 10^{10(\mathrm{c})}$ \\
\hline $\mathrm{C}_{12}\left(\mathrm{~N} / \mathrm{m}^{2}\right)$ & $4.98 \times 10^{10(\mathrm{c})}$ & $6.66 \times 10^{10(c)}$ \\
\hline $\mathrm{E}_{\mathrm{g}}(\mathrm{eV})$ & $2.821^{\text {(a) }}$ & $3.840^{(b)}$ \\
\hline $\mathrm{m}_{\mathrm{e}}$ & $0.16^{(a)}$ & $0.27^{(\mathrm{d})}$ \\
\hline $\mathrm{m}_{\mathrm{HH}}$ & $0.6^{(\mathrm{d})}$ & $0.49^{(\mathrm{d})}$ \\
\hline$m_{L H}$ & $0.16^{(\mathrm{e})}$ & $0.4^{(\mathrm{e})}$ \\
\hline$\alpha$ & $-4.25^{(f)}$ & $-4.53^{(\mathrm{g})}$ \\
\hline $\mathrm{b}(\mathrm{eV})$ & $-1.2^{(\mathbf{a})}$ & $-1.25^{(a)}$ \\
\hline$\Delta_{\mathrm{SO}}(\mathrm{eV})$ & $0.43^{(a)}$ & $0.07^{(\mathrm{d})}$ \\
\hline
\end{tabular}

(a) Ref. 9

(b) From thermal expansion coefficients

$\begin{array}{lll}\text { (c) Ref. } 5 & \text { (d) } \text { Ref. } 10 & { }^{\text {(e) }} \text { Ref. } 11\end{array}$

${ }^{\text {(f) }}$ Ref. $12 \quad{ }^{\text {(g) }}$ Ref. 13

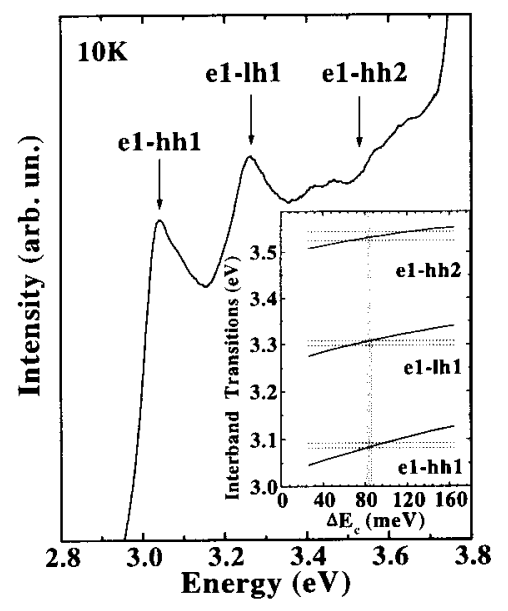

Fig. 1 Absorption spectrum of the $\mathrm{ZnSe} / \mathrm{ZnS}$ SLs. Inset: Interband transitions to evaluate the $\mathrm{CB}$ offset.

The inset of Fig. 1 shows the calculated interband transitions as a function of the CB discontinuity. The continuous lines represent the calculated transitions energies, while the dashed horizontal lines indicates the energy intervals of the corresponding transitions in the absorption spectrum of Fig. 1. The spectral broadening of the absorption curve is indicated by the energy separation of the dashed lines. The vertical gray band represents the $\mathrm{CB}$ values that fit (within the experimental errors) the experimental data. From these results a CB offset of $83 \pm 2 \mathrm{meV}$ can be derived, which corresponds to a $8.4 \%$ of the total band offset in the CB. Fig. 2 shows the calculated MQW band structure resulting from the above calculations. $\mathrm{V}_{\mathrm{CB}}, \mathrm{V}_{\mathrm{VB}}(\mathrm{HH})$ and $\mathrm{V}_{\mathrm{VB}}(\mathrm{LH})$ represent the $\mathrm{CB}$ and $\mathrm{VB}$ offsets, respectively. Electron (e), heavy-hole (hh) and light-hole (lh) energy levels in the CB and $\mathrm{VB}$, respectively are indicated. The arrows in the inset of Fig. 1 indicate the electronic transitions corresponding to the thus calculated excitonic peak positions superposed to the experimental absorption spectrum. We can thus ascribe the two peaks at $3.048 \mathrm{eV}$ and $3.260 \mathrm{eV}$ in the absorption spectrum as the $n=1$ electron-to-hh and electron-to-lh transitions, respectively.

Finally, the calculated interband transition energies as a function of the well width are shown in Fig. 3 . These results represents the envelope-function calculations in the effective-mass approximation for well width values ranging between $5 \mathrm{ML}$ and $21 \mathrm{ML}$, and assuming a fully pseudomorphic SLs. In this case the value of total strain in the $\mathrm{ZnSe}$ wells is equal to $-4.40 \times 10^{-2}$ and it amounts to $+1.48 \times 10^{-3}$ in the $\mathrm{ZnS}$ barriers. 


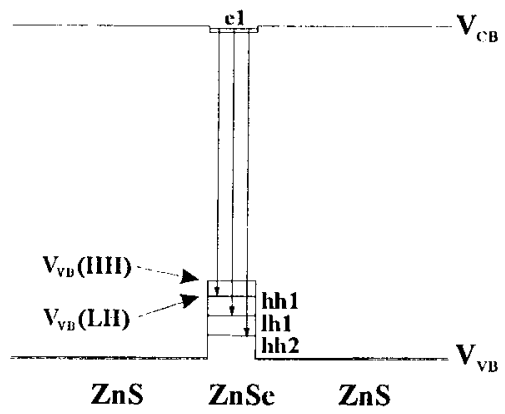

Fig 2: Schematic of the valence and conduction band alignments for the measured $\mathrm{ZnSe} / \mathrm{ZnS}$ SLs (10K). The clectronic transitions are also shown by arrows.

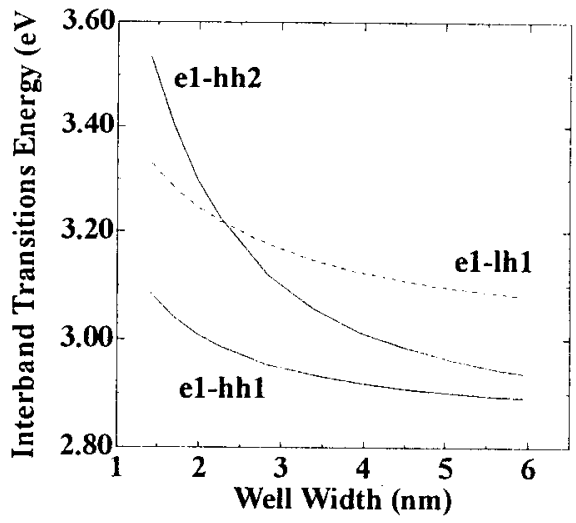

Fig. 3: $10 \mathrm{~K}$ Interband transition energies as a function of the $\mathrm{ZnSe}$ well width for a fully pseudomorphic $\mathrm{ZnSe} / \mathrm{ZnS}$ SL.

\section{Conclusions}

A detailed calculation of the effects of lattice and thermal strains inside nearly pseudomorphic shortperiod $\mathrm{ZnSe} / \mathrm{ZnS}$ SLs on the heterostructure electronic states was reported. Comparison was then made with experimental electronic transitions derived from $10 \mathrm{~K}$ absorption spectroscopy measurements performed on a partially relaxed 30 periods $\mathrm{ZnSe} / \mathrm{ZnS} \mathrm{SL}$. The intrinsic conductionto-valence band offset ratio was finally found to be $8: 92$ for this type of SL structure.

\section{References}

[1] R. Cingolani, P. Prete, M. Lomascolo, G. Coli', L. Calcagnile, and N. Lovergine, Appl. Phys. Lett. 70 (1997), p. 2943

[2] N. Lovergine, P. Prete, G. Leo, L. Calcagnile, R. Cingolani, A. M. Mancini, F. Romanato, and A.V. Drigo, Cryst. Res. Technol. 33 (1998), p. 183

[3] R. Cingolani, P. Prete, D. Greco, P.V. Giugno, M. Lomascolo, R. Rinaldi, L. Calcagnile, L. Vanzetti, L. Sorba, and A. Franciosi, Phys. Rev. B 51 (1995), p. 5176

[4] Y. Kawakami, T. Taguchi, and A. Hiraki, J. Cryst. Growth 93 (1988), p. 714.

[5] Taguchi, Y. Kawakami, Y. Yamada, Physica B 191 (1993).

[6] G. Leo, L. Lazzarini, Lovergine, F. Romanato, A.V. Drigo, J. Cryst- Growth 173 (1997), p. 277.

[7] B. Gil, T. Cloitre, M. Di Blasio, P. Bigenwald, L. Aigouy, N. Briot, O. Briot, D. Bouchara, R.L. Aulombard, and J. Calas, Phys. Rev. B 50 (1994), p. 18231.

[8] F. H. Pollak and M. Cardona, Phys. Rev. 172 (1968), p. 816.

[9] Numerical Data and Functional Relationship in Science and Technology, edited by 0 . Madelung, Landolt-Bornstein, New Series, Group III, Vol. 17 (Springer, Berlin, 1982).

[10] K. Shahzad and D. Olego, Phys. Rev. B 38 (1988), p. 1417.

[11] P. Lawaetz, Rev. B 4 (1971), p. 3460.

[12] D. W. Langer,R. N. Euwema, K. Era, and T. Koda, Rev. B 2 (1970), p. 4005

[13] S. Ves, U. Schwarz, N.E. Christensen, K. Syassen, and M. Cardona, Phys. Rev. B 42 (1990), p. 9113. 
Ultrafast Phenomena in Semiconductors

10.4028/www.scientific.net/MSF.297-298

Lattice Strain and Band Offset Determination in ZnSe/ZnS Short-Period Superlattices Grown by MOVPE on (100)GaAs

10.4028/www.scientific.net/MSF.297-298.265 